Ekaterina Kovacheva ${ }^{\dagger \star}$, Lukas Baron ${ }^{\dagger}$, Steffen Schuler, Tobias Gerach, Olaf Dössel, and Axel Loewe

\title{
Optimization Framework to Identify Constitutive Law Parameters of the Human Heart
}

https://doi.org/10.1515/cdbme-2020-3025

\begin{abstract}
Over the last decades, computational models have been applied in in-silico simulations of the heart biomechanics. These models depend on input parameters. In particular, four parameters are needed for the constitutive law of Guccione et al., a model describing the stress-strain relation of the heart tissue. In the literature, we could find a wide range of values for these parameters.

In this work, we propose an optimization framework which identifies the parameters of a constitutive law. This framework is based on experimental measurements conducted by Klotz et al.. They provide an end-diastolic pressure-volume relationship. We applied the proposed framework on one heart model and identified the following elastic parameters to optimally match the Klotz curve: $C=313 \mathrm{~Pa}, b_{f}=17.8, b_{t}=7.1$ and $b_{f t}=12.4$.

In general, this approach allows to identify optimized parameters for a constitutive law, for a patient-specific heart geometry. The use of optimized parameters will lead to physiological simulation results of the heart biomechanics and is therefore an important step towards applying computational models in clinical practice.
\end{abstract}

Keywords: Optimization, Passive force, Material properties, Myocardial stiffness, Klotz curve

\section{Introduction}

The human heart is a complex organ, involving the interaction of different phenomena. On the one hand, blood is flowing through the heart chambers and applies pressure on the endocardial surfaces. On the other hand, electrical waves propagate through the myocardium to activate a contraction force, which - added to the passive force - works against the chamber pres-

${ }^{*}$ Corresponding author: Ekaterina Kovacheva ${ }^{\dagger}$, Institute of Biomedical Engineering, Karlsruhe Institute of Technology (KIT), Kaiserstr. 12, 76131 Karlsruhe, Germany, e-mail: publications@ibt.kit.edu

Lukas Baron ${ }^{\dagger}$, Steffen Schuler, Tobias Gerach, Olaf Dössel, Axel Loewe, Institute of Biomedical Engineering, Karlsruhe Institute of Technology (KIT), 76131 Karlsruhe, Germany. ${ }^{\dagger}$ These authors contributed equally. sure and results in the deformation of the myocardium. Those phenomena are described by different mathematical models, which are implemented in a numerical framework in order to simulate the human heart beat in-silico. Such simulations could be used by clinicians to indicate cardiac pathologies.

In particular, the myocardial passive force, which arises from the intrinsic material properties of the heart tissue, can be described by the commonly applied constitutive law of Guccione et al. [1]. The values of the input parameters have to be set prior to the simulation. However, a wide range of values can be found but in the literature, e.g., between $189 \mathrm{~Pa}$ and $3000 \mathrm{~Pa}$ for the $C$ parameter [2].

Dabiri et al. [3], Genet et al. [4] and Palit et at. [5] estimated the material properties based on distances to an enddiastolic pressure-volume curve proposed by Klotz et al. [6] Asner et al. [7] estimated the material properties using deformation data, in particular in the diastolic phase. Balaban et al. also estimated the elastic parameters via constrained minimization and adjoint gradient calculation [8].

In this work, we present an optimization framework which identifies the parameters of a constitutive law for a certain heart geometry. We estimated the parameter which scales the global stiffness $(C)$ and three further parameters to scale the stiffness in fiber $\left(b_{f}\right)$, sheet normal $\left(b_{t}\right)$ and sheet $\left(b_{f t}\right)$ direction. The objective function is based on measured enddiastolic pressure-volume relations (EDPVR) as described in Klotz et al., which are characterized by the passive behaviour of the tissue [6]. Furthermore, a target ratio of pressure-free and inflated ventricle volume is part of the objective function for the optimization. We applied the proposed framework on one clinical heart model and identified the parameters of the constitutive law.

\section{Methods}

The presented optimization framework aims at identifying the optimized parameters of a constitutive law for a given patientspecific geometry. In Figure 1, the optimization workflow is schematically presented and described in the following. 


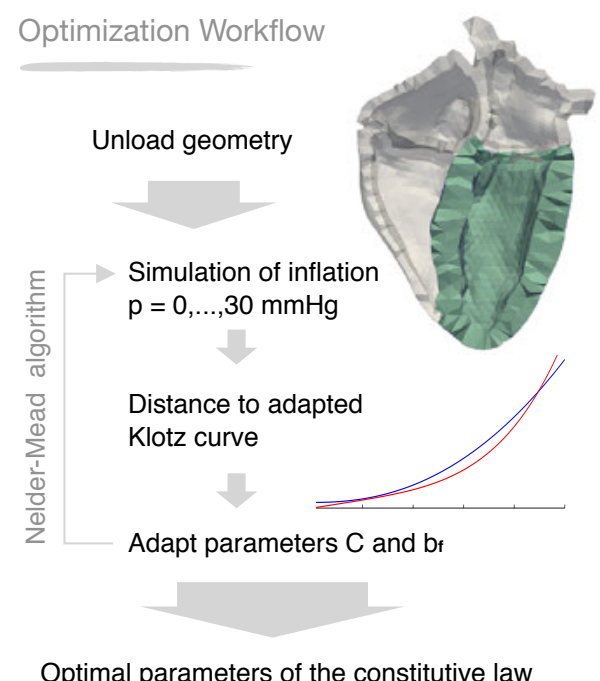

Fig. 1: The optimization workflow is schematically presented. The unloaded geometry is visualized, clipped along the long axis, the left ventricle is colored in green.

\subsection{Optimization}

The objective function of the minimization procedure is defined based on the EDPVR. Klotz et al. measured this relation in 80 ex-vivo human hearts of different etiologies [6] by applying pressure between 0 and $30 \mathrm{mmHg}$ in the left ventricle and simultaneously measuring the volume of the cavity. For every ventricle, they normalized the volume to range between 0 and 1 and provided one exponential curve approximating all measurements with a single equation: $P=28.2 \bar{V}^{2.79} \mathrm{mmHg}$, where $\bar{V}$ is the normalized volume and $P$ is the corresponding ventricular pressure [6].

We adapted the exponential expression by adding a linear term in order to allow a non-zero derivative at the start point $(0,0 \mathrm{mmHg})$. We fitted the equation coefficients to the data measured by Klotz et al.. Furthermore, we imposed a regression condition for both start and end points of the curve $((0,0 \mathrm{mmHg})$ and $(1,30 \mathrm{mmHg}))$, since the simulated curve will certainly pass through these points due to the definition of volume normalization. For the adapted Klotz curve we obtained the following expression:

$$
P^{a d}=7.015 \bar{V}+22.985 \bar{V}^{4.322} \mathrm{mmHg} .
$$

Additionally, Klotz et al. provided a formula describing the volume ratio for different pressure values:

$$
V_{0}=V_{m}\left(0.6-0.006 P_{m} \mathrm{mmHg}^{-1}\right),
$$

where $V_{m}$ is the left ventricular cavity volume at pressure $P_{m}$ and $V_{0}$ is the initial volume (at pressure $0 \mathrm{mmHg}$ ) [6]. Therefore, we aim at volume ratio $V_{30} / V_{0}$ of 2.38 , which is a part of the objective function for the optimization.
The objective function is defined as the sum of the squared distances between the adapted Klotz curve and a curve relating the normalized volume and the applied pressure in a numerical simulation. Additionally, a weight term is added, which considers the deviation from the target volume ratio:

$$
F^{o b j}=\sum_{i=1}^{K}\left(P_{i}^{s i m}-P_{i}^{a d}\right)^{2}+\lambda\left|\frac{V_{30}}{V_{0}}-2.38\right|
$$

where $P^{s i m}$ is the pressure applied in the simulation, the index $i$ samples equidistantly the range of the volume values, $K=$ 100 and the weight $\lambda$ is set also to 100 .

The Nelder-Mead simplex algorithm is applied to minimize the objective function [9]. It is a gradient-free direct search method and is suited for nonlinear optimization problems. We used its implementation in SciPy [10].

\subsection{Geometrical Model}

In this work, we applied the proposed optimization framework to a human heart geometry comprising four chambers: two atria and two ventricles. The geometry of the heart was determined from clinical volumetric magnetic resonance images of the whole heart in the end-diastolic state. Since at enddiastole, the pressure in the left ventricle is about $8 \mathrm{mmHg}$, an unloaded state for the obtained geometry needs to be estimated. That is the state at zero pressure and is obtained by the unloading algorithm proposed by Bols et al. [11] (with parameter set $C=324 \mathrm{~Pa}, b_{f}=8, b_{t}=3.2$ and $b_{f t}=5.6$ ). The unloaded state is the initial geometry for the simulation of the inflation (pressure application).

The heart geometry was spatially discretized by 21069 tetrahedral finite elements (with quadratic shape functions) and 38232 nodes. Additionally, the endocardial surface of the left ventricle was defined by 6376 triangles (with linear shape functions). The fiber orientation in the ventricles was calculated with the algorithm proposed by Bayer et al. [12].

\subsection{Simulation of Inflation}

To simulate the inflation of the left ventricle of the heart, we used the numerical simulator CardioMechanics described in Fritz et al. [13]. CardioMechanics implements the finite element method to solve the equation for balance of linear momentum. It provides the deformation resulting from the balance of the internal and external forces. The latter are arising from pressure on the endocardial surface. The inflation of the left ventricle was simulated by applying pressure increasing linearly from $0 \mathrm{mmHg}$ to $30 \mathrm{mmHg}$ over $1 \mathrm{~s}$. The passive in- 
ternal forces arise from the constitutive law, which relates the stress and the strain of the tissue.

We applied the model of Guccione et al. [1] describing hyperelastic, transversely isotropic material properties. Furthermore, near incompressibility was imposed by adding a penalty term to the strain energy function:

$$
\begin{aligned}
W & =\frac{C}{2}\left(e^{Q}-1\right)+\frac{K}{2}(\operatorname{det}(F)-1)^{2}, \\
Q & =b_{f} E_{11}^{2}+b_{t}\left(E_{22}^{2}+E_{33}^{2}+E_{23}^{2}+E_{32}^{2}\right) \\
& +b_{f t}\left(E_{12}^{2}+E_{21}^{2}+E_{13}^{2}+E_{31}^{2}\right),
\end{aligned}
$$

where $C, b_{f}, b_{t}$ and $b_{f t}$ are the parameters of the Guccione et al. model, $E_{i j}(i, j \in[1,2,3])$ are elements of the Green-strain tensor, $\operatorname{det}(F)$ is the determinant of the deformation tensor and $K=2 \cdot 10^{5} \mathrm{~Pa}$ scales the penalty term. To reduce the dimension of the optimization space, we coupled the parameters as proposed by Genet et al. [4]: $b_{t}=0.4 b_{f}$ and $b_{f t}=0.7 b_{f}$. Therefore, only the parameters $C$ and $b_{f}$ from equation (4) need to be identified. For both ventricles the same parameter set was used.

\section{Results}

To include measured EDPVR in the objective function, we adapted the Klotz curve prior to the optimization. In Figure 2, the measured relations of pressure and normalized volume, as described in Klotz et al. [6], are marked by a plus. The adapted as well as the original Klotz curve are plotted, respectively in red and cyan color. The red curve was used in the objective function. Note, that the adapted Klotz curve yields a better fit for the measured data for pressures smaller than $10 \mathrm{mmHg}$ (the squared distance is $18.02 \mathrm{mmHg}$ ) compared to original Klotz curve (the squared distance is $22.43 \mathrm{mmHg}$ ). Pressures smaller than $10 \mathrm{mmHg}$ are particularly relevant for description of the passive mechanical function during diastole.

We applied the optimization framework and identified the following values for the parameters of the Guccione et al. constitutive law: $C=313 \mathrm{~Pa}, b_{f}=17.8, b_{t}=7.1$ and $b_{f t}=12.4$. This is the optimized parameter set for the described four chamber geometry model of the heart.

The inflation simulation of the left ventricle starts with an unloaded state of the geometry (at zero pressure) and provides the inflated state (at pressure $30 \mathrm{mmHg}$ ). Both states are visualized in Figure 3, the inflated state is obtained with the optimized parameter set. During the inflation, the left ventricle volume increased from $107 \mathrm{ml}$ to $264 \mathrm{ml}$ leading to a volume ratio of 2.45. A thinning of the myocardial wall is evident. The pressure-volume relation of the inflation simulation with the

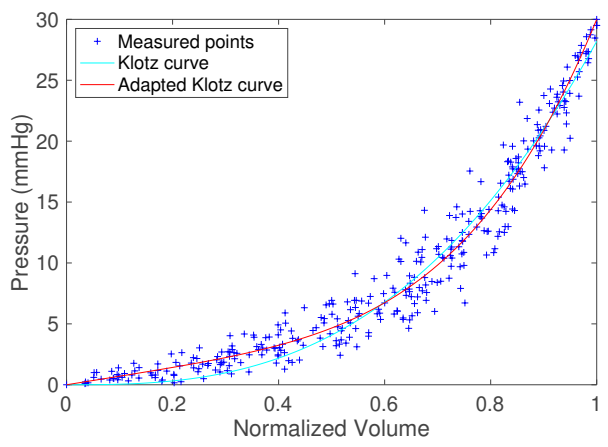

Fig. 2: The blue crosses represent measurements by Klotz et al., the cyan line is the Klotz curve [6] and the red curve is the adapted Klotz curve.
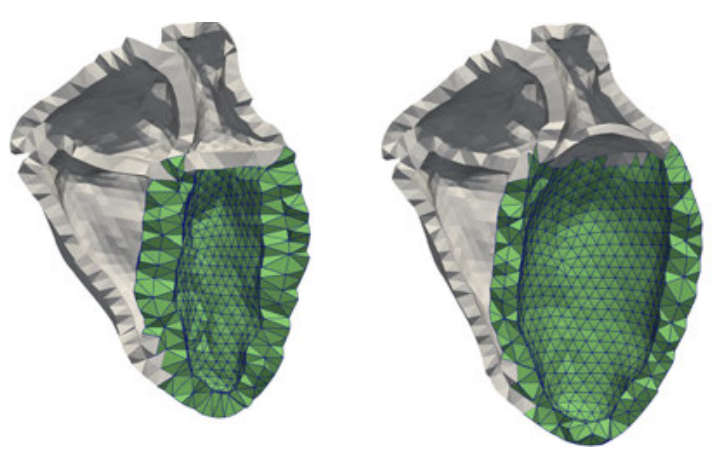

Fig. 3: Left: unloaded state of the geometry clipped along the long axis. Right: the inflated state at pressure of $30 \mathrm{mmHg}$. The left ventricle is highlighted with green colour.

optimized parameter set is visualized in Figure 4. As a comparison, the adapted Klotz curve is also plotted.

\section{Discussion}

In this work, we identified a parameter set for the constitutive law of Guccione et al. for a patient-specific anatomical model by solving an optimization problem. The simulated Klotz curve is close to the adapted Klotz curve and the volume ratio differs from the target ratio by only 0.07 . The identified parameter set lies within the value range found in literature.

We examined further parameter sets and compared the corresponding simulated curve to the adapted Klotz curve. For example, for $C=770 \mathrm{~Pa}, b_{f}=2.2$, we obtained a better match of the two curves. Nevertheless, the volume ratio was 6.4, which is far from the target ratio 2.38. The choice of the weight factor $\lambda$ in the objective function could be crucial as it determines the balance of two objectives. The value of the target ratio was derived from a linear relation as proposed by Klotz et al. [6]. This relation was established based on measurements, which appear to lie within a circular region rather 


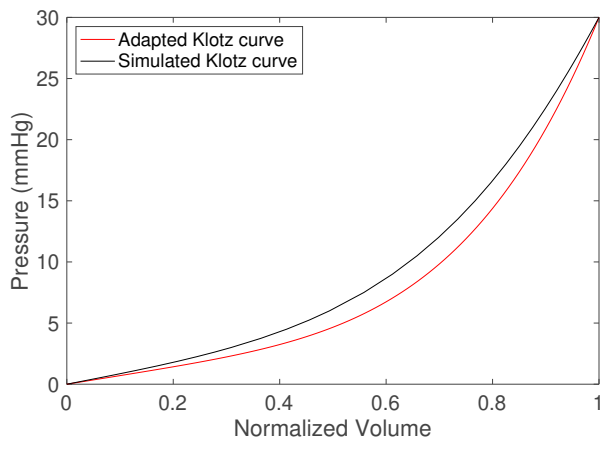

Fig. 4: The red curve is the adapted Klotz curve and the black one is calculated based on the simulation result for the optimized parameter set.

than on a line. Therefore, a more reasonable term to add to the objective function may exist.

The unloaded state depends on the parameters of the constitutive law and therefore, should be calculated for each set of parameters. In this work, we simplified the process by calculating this state once, prior to the optimization and used the same unloaded state in all inflation simulations. Since the unloaded state of the geometry was obtained with a parameter set which is very close to the optimized set, we assume this simplification does not change the result considerably.

The proposed framework can be applied to further constitutive laws, as far as they are implemented in the numerical framework which simulates the inflation. Also, it can be applied on any geometry with a closed cavity. The proposed workflow regards only global passive behaviour and does not compare to any local measurements such as strain and stretch of the tissue.

To the best of our knowledge, there is currently no way to validate the obtained parameters for a patient-specific geometry. Therefore, we are aiming at developing an alternative method to estimate material properties and compare them to the proposed parameters in this work. This might include patient specific clinical measurements, such as cine magnetic resonance tomography (MRI) or tagged MRI.

\section{Conclusion}

The presented optimization framework can identify parameters for the constitutive law underlying numerical simulations of the heart's biomechanics. Therefore, in simulations we are able to use a physiological stress-strain relation of the myocardial tissue, which leads to more realistic deformation of the heart tissue. With our framework we could identify optimized parameters for one patient-specific heart model. The presented framework can also be applied to further patient-specific heart geometries and other constitutive laws. Altogether, the application of optimized parameters in a numerical simulation can lead to more physiological deformation of the heart. Therefore, it is an important step towards applying computational models in clinical practice.

\section{Author Statement}

Research funding: We gratefully acknowledge funding by the Deutsche Forschungsgemeinschaft (DFG, German Research Foundation) - Project-ID 258734477 - SFB 1173, HEiKA Heidelberg Karlsruhe Research Partnership, Heidelberg University, Karlsruhe Institute of Technology (KIT), Germany and the Federal Ministry of Education and Research, Germany, Grant Number: 05M2016 . Conflict of interest: Authors state no conflict of interest. Informed consent: Informed consent has been obtained from all individuals included in this study. Ethical approval: The research related to human use complies with all the relevant national regulations, institutional policies and was performed in accordance with the tenets of the Helsinki Declaration, and has been approved by the authors' institutional review board or equivalent committee.

\section{References}

[1] J. M. Guccione, L. K. Waldman, J. Biomechanical Engineering 1991, 113, 42-55.

[2] S. Kallhovd, J. Sundnes, S. T. Wall, Computer methods in biomechanics and biomedical engineering 2019, 22, 664675.

[3] Y. Dabiri, J. Guccione, Journal of biomechanical engineering 2019, DOI 10.1115/1.4044215.

[4] M. Genet, J. M. Guccione, Journal of applied physiology (Bethesda Md. : 1985) 2014, 117, 142-52.

[5] A. Palit, M. A. Williams, Medical \& biological engineering \& computing 2018, 56, 1615-1631.

[6] S. Klotz, D. Burkhoff, American journal of physiology. Heart and circulatory physiology 2006, 291, H403-12.

[7] L. Asner, D. Nordsletten, Biomechanics and modeling in mechanobiology 2016, 15, 1121-39.

[8] G. Balaban, H. Finsberg, M. E. Rognes, Biomechanics and modeling in mechanobiology 2018, 17, 1317-1329.

[9] F. Gao, L. Han, Computational Optimization and Applications 2010, 51, 259-277.

[10] P. Virtanen, P. van Mulbregt, S. 1. 0. Contributors, Nature Methods 2020.

[11] J. Bols, J. Vierendeels, Journal of Computational and Applied Mathematics 2013, 246, 10-17.

[12] J. D. Bayer, N. A. Trayanova, Annals of Biomedical Engineering 2012, 40, 2243-2254.

[13] T. Fritz, O. Dössel, Biomechanics and Modeling in Mechanobiology 2014, 13, 627-641. 